\title{
Corrigendum: Sho1 and Msb2 Play Complementary but Distinct Roles in Stress Responses, Sexual Differentiation, and Pathogenicity of Cryptococcus neoformans
}

\author{
Yee-Seul So ${ }^{1 \dagger}$, Juyeong Jang ${ }^{1 \dagger}$, Goun Park ${ }^{1 \dagger}$, Jintao $X u^{2}$, Michal A. Olszewski ${ }^{2,3}$ and \\ Yong-Sun Bahn ${ }^{1 *}$
}

\begin{abstract}
${ }^{1}$ Department of Biotechnology, College of Life Science and Biotechnology, Yonsei University, Seoul, South Korea, ${ }^{2}$ Division of Pulmonary and Critical Care Medicine, Department of Internal Medicine, University of Michigan Medical School, Ann Arbor,
\end{abstract} MI, United States, ${ }^{3}$ VA Medical Center Ann Arbor Research Service, Ann Arbor, MI, United States

Keywords: HOG, mucin, C. neoformans, mating, osmotic stress

\section{A Corrigendum on}

OPEN ACCESS

Edited by:

Gustavo Henrique Goldman,

University of São Paulo, Brazil

Reviewed by:

Iran Malavazi,

Federal University of São Carlos, Brazil

*Correspondence:

Yong-Sun Bahn

ysbahn@yonsei.ac.kr

tThese authors have contributed equally to this work

Specialty section:

This article was submitted to

Fungi and Their Interactions,

a section of the journal

Frontiers in Microbiology

Received: 06 July 2020

Accepted: 24 July 2020

Published: 24 September 2020

Citation:

So Y-S, Jang J, Park G, XU J, Olszewski MA and Bahn Y-S (2020)

Corrigendum: Sho1 and Msb2 Play

Complementary but Distinct Roles in

Stress Responses, Sexual

Differentiation, and Pathogenicity of

Cryptococcus neoformans.

Front. Microbiol. 11:1956

doi: 10.3389/fmicb.2020.01956
Sho1 and Msb2 Play Complementary but Distinct Roles in Stress Responses, Sexual Differentiation, and Pathogenicity of Cryptococcus neoformans

by So, Y.-S., Jang, J., Park, G., Xu, J., Olszewski, M. A., and Bahn, Y.-S. (2018). Front. Microbiol. 9:2958. doi: 10.3389/fmicb.2018.02958

We recently found that the phospho-p44/42 antibody (Cell Signaling Technology) used in the original article is not specific to the phosphorylated form of Cpk1 in Cryptococcus neoformans. Therefore, we removed Cpk1 phosphorylation-related content and Figures (Figures 7D, 8A) and corrected the original article, as shown below.

A correction has been made to the Abstract.

The high-osmolarity glycerol response (HOG) pathway is pivotal in environmental stress response, differentiation, and virulence of Cryptococcus neoformans, which causes fatal meningoencephalitis. A putative membrane sensor protein, Sho1, has been postulated to regulate HOG pathway, but its regulatory mechanism remains elusive. In this study, we characterized the function of Shol with relation to the HOG pathway in C. neoformans. Shol played minor roles in osmoresistance, thermotolerance, and maintenance of membrane integrity mainly in a HOG-independent manner. However, it was dispensable for cryostress resistance, primarily mediated through the HOG pathway. A mucinlike transmembrane (TM) protein, Msb2, which interacts with Sho1 in Saccharomyces cerevisiae, was identified in C. neoformans, but found not to interact with Sho1. MSB2 codeletion with SHO1 further decreased osmoresistance and membrane integrity, but not thermotolerance, of sho1 $\Delta$ mutant, indicating that both factors play to some level redundant but also discrete roles in C. neoformans. Sho1 and Msb2 played redundant roles in promoting the filamentous growth in sexual differentiation in a Cpk1-independent manner, in contrast to the inhibitory effect of the HOG pathway in the process. Both factors also played redundant roles in maintaining cell wall integrity in the absence of Mpk1. Finally, Sho1 and Msb2 play distinct but complementary roles in the pulmonary virulence of C. neoformans. Overall, Sho1 and Msb2 play complementary but distinct roles in stress response, differentiation, and pathogenicity of C. neoformans.

Corrections have been made to the Materials and Methods section, sub-section Western Blot Analysis for the Hog1 Phosphorylation. 


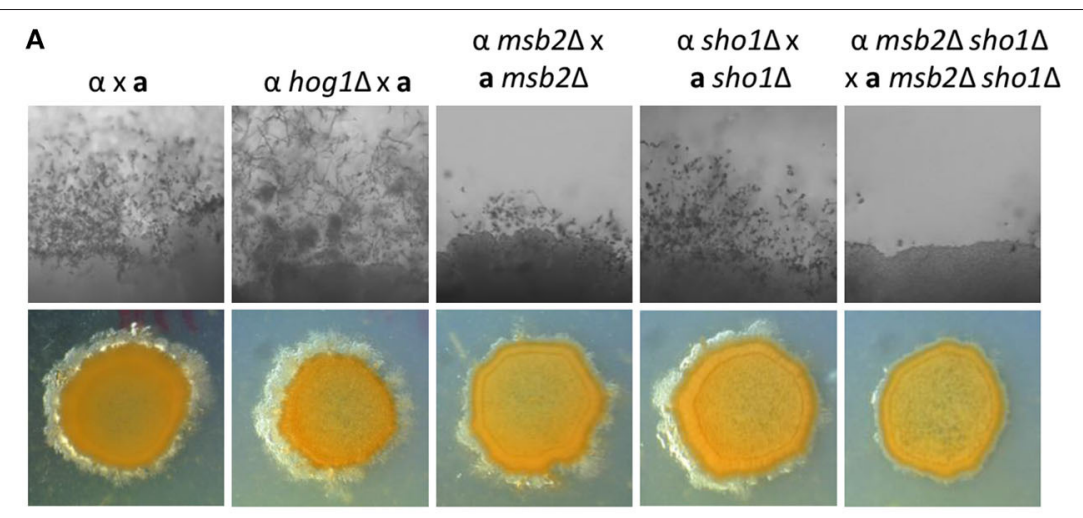

B $\alpha \times a$

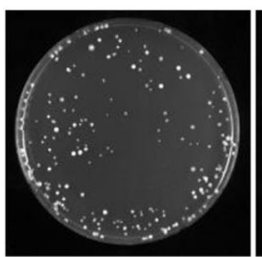

$100 \%$

$$
\alpha \operatorname{sho1\Delta x}
$$
a sho1 $\Delta$

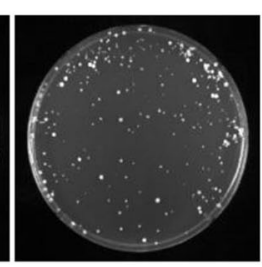

$102.7 \pm 4.6 \%$

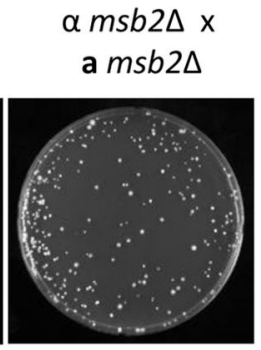

$112.5 \pm 6.8 \%$

FIGURE 7 | Sho1 and Msb2 play redundant positive roles in the filamentous growth of $C$. neoformans. (A) Opposite mating type (MAT $\alpha$ and MATa) cells were incubated for $16 \mathrm{~h}$ in YPD liquid medium at $30^{\circ} \mathrm{C}$. Opposite mating type cells were mixed at equal concentration (10 $\left.{ }^{7} \mathrm{cells} / \mathrm{mL}\right)$, spotted $(5 \mu \mathrm{L})$ on $\mathrm{V} 8 \mathrm{medium}$, and further incubated in the dark at room temperature for 2 weeks. This mating experiment was repeated twice and one representative image was shown here. (B) Mixed opposite mating type cells were spotted on V8 medium and incubated for 1 day at room temperature in the dark. After cells were grown on V8 medium, the cells were resuspended in 1-mL dH $\mathrm{H}_{2} \mathrm{O}$ and diluted to 1/100. Then, $200 \mu \mathrm{L}$ of the suspension was spread on YPD medium containing nourseothricin and G418. The plates were further incubated at $30^{\circ} \mathrm{C}$ and colonies were counted. (C) The northern blot analysis was performed with total RNAs from strains grown on V8 medium for $18 \mathrm{~h}$. The northern blot membrane was hybridized with the mating pheromone-gene (MF 1 )-specific probe. This northern blot analysis was repeated twice and one representative result was shown here.

\section{"Western Blot Analysis for the Hog1 Phosphorylation.}

Each strain was grown in 50-mL YPD medium at $30^{\circ} \mathrm{C}$ for $16 \mathrm{~h}$. Then, the overnight culture was inoculated into fresh YPD medium and, then, incubated for about $4 \mathrm{~h}$ at $30^{\circ} \mathrm{C}$ to the OD600 of 0.6 . A $50 \mathrm{~mL}$ of the liquid culture was used at each stress time point. At various time points after the stress, $50 \mathrm{~mL}$ of cell suspension was mixed with equal volume of ice-cold stop solution $(0.9 \% \mathrm{NaCl}, 1 \mathrm{mM} \mathrm{NaN3}, 10 \mathrm{mM}$ EDTA, and $50 \mathrm{mM}$ $\mathrm{NaF}$ ). The cells were harvested at $3000 \mathrm{rpm}$ at $4^{\circ} \mathrm{C}$ for $5 \mathrm{~min}$ and, then, washed once in ice-cold stop solution. The cell pellet was resuspended in the lysis buffer $(50-\mathrm{mM}$ Tris- $\mathrm{HCl} \mathrm{pH} 7.5$, $1 \%$ sodium deoxycholate, $5-\mathrm{mM}$ sodium pyrophosphate, $10-\mathrm{nM}$ sodium orthovanadate, $50-\mathrm{mM} \mathrm{NaF}, 0.1 \%$ SDS, and $1 \%$ Triton $\mathrm{X}-100)$ containing protease inhibitor cocktail (Calbiochem) and disrupted with 0.5-mm zirconia/silica beads (BioSpec Products, Inc.). After collecting the cell lysates, protein concentrations were determined using the Pierce BCA Protein Assay Kit (Thermo Scientific), and an equal amount of protein was loaded into a $10 \%$ SDS-PAGE gel and transferred to Immunoblot PVDF membrane (Bio-Rad). For detecting the phosphorylated forms of Hog1, we used phospho-p38 MAPK antibody (Cell Signaling Technology).
In addition, anti-Hog1 antibody (Santa Cruz Biotechnology, SC2004) was used as a loading control. Secondary antibody used was goat anti-rabbit immunoglobulin $\mathrm{G}$ peroxidase-conjugated (Santa Cruz Biotechnology, SC-2004) and the blot was developed using the ECL solution."

A correction has been made to the Materials and Methods section, sub-section Mating, Cell Fusion, and Pheromone Gene Expression Assay.

"For analyzing mating phenotypes opposite mating type (MAT $\alpha$ and MATa) cells were cultured in YPD medium at $30^{\circ} \mathrm{C}$ for $16 \mathrm{~h}$ and equal concentration of cells $\left(10^{7}\right.$ cells $\left./ \mathrm{mL}\right)$ were mixed, spotted onto V8 mating media ( $\mathrm{pH} 5)$, and incubated in the dark at room temperature for 1-2 weeks. The filamentous growth was monitored and photographed using an Olympus BX51 microscope equipped with a SPOT Insight digital camera. For the cell fusion assay, the concentration of cells was adjusted to $10^{7}$ cells $/ \mathrm{mL}$ with phosphate-buffered saline. Each MAT $\alpha$ and MATa strain was mixed in an equal volume, spotted onto a V8 medium, and incubated in the dark at room temperature for $24 \mathrm{~h}$. Then, the cells were scraped, resuspended in 1- $\mathrm{mL}$ distilled water, and spread onto YPD medium containing both nourseothricin 


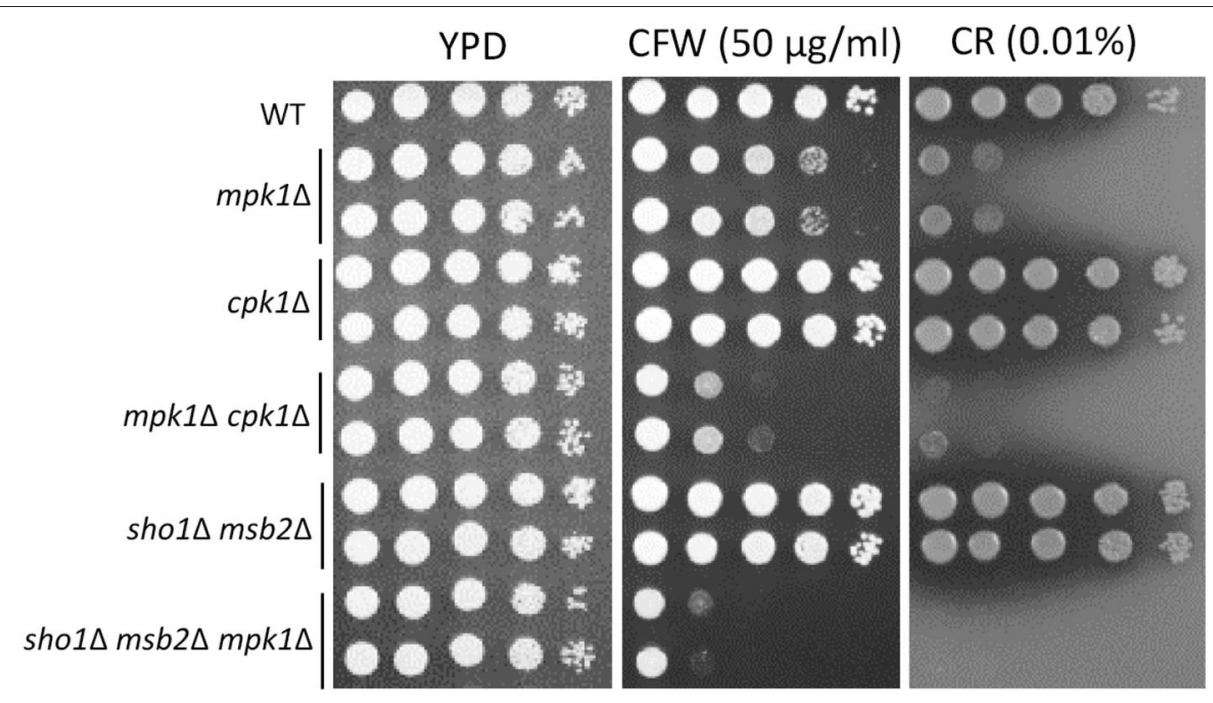

FIGURE 8 | Sho1 and Msb2 have a redundant role in regulating cell wall integrity. Wild-type (WT; H99), mpk1 $\Delta$ (YSB3814 and YSB3816), cpk1 $\Delta$ (YSB127 and YSB128), mpk1 $\Delta$ cpk1 $\Delta$ (YSB6089 and YSB6091), sho1 $\Delta$ msb2 $\Delta$ (YSB3605 and YSB3606), and sho1 $\Delta$ msb2 $\Delta$ mpk1 $(Y S B 6675$ and YSB6676). Each strain was grown overnight at $30^{\circ} \mathrm{C}$ in YPD medium, 10-fold serially diluted, and spotted onto YPD medium containing the indicated concentrations of Congo red (CR) and calcofluor white (CFW). The plates were further incubated for 2-3 days and photographed. This spot assay was repeated more than three times and one representative image was shown here.

$(100 \mu \mathrm{g} / \mathrm{mL})$ and $\mathrm{G} 418(50 \mu \mathrm{g} / \mathrm{mL})$. The plates were further incubated at $30^{\circ} \mathrm{C}$, and the number of colonies was counted. For monitoring the pheromone gene expression, the MAT $\alpha$ and KN99a strains were mixed with an equal concentration of cells $\left(10^{8}\right.$ cells $\left./ \mathrm{mL}\right)$, spread onto the V8 medium, and incubated in the dark at room temperature for 18 or $24 \mathrm{~h}$. Then, cells were scraped, pelleted, frozen in liquid nitrogen, and lyophilized overnight for the total RNA isolation, followed by the northern blot analysis with the specific mating pheromone gene $(M F \alpha 1)$ specific probe."

A correction has been made to the Results section, sub-section Sho1 and Msb2 Play Redundant Roles in the Filamentation Process of C. neoformans, Paragraph 2.

We monitored pheromone expression levels under the unilateral and bilateral mating setup among sho1 $\Delta, m s b 2 \Delta$, and $s h o 1 \Delta, m s b 2 \Delta$ mutants compared with the WT strain to determine which stage of mating is regulated by Shol and Msb2. We observed that the pheromone-gene expression was as markedly induced in the sho1 $\Delta, m s b 2 \Delta$, and sho1 $\Delta m s b 2 \Delta$ mutants as WT when $\alpha$ cells were cocultured with a cells (Figure 7C). These findings suggested that Sho1 and Msb2 play complementary positive roles in the late stage (filamentation), but not the early stage (pheromone expression and cell fusion), of mating in C. neoformans.

The corrected Figure 7 appears below.

Corrections have been made to the Results section, subsection The Role of Cpk1, Msb2 and Sho1 in the Cell-Wall Integrity of C. neoformans, Paragraph 1.

The Role of Cpk1, Msb2 and Sho1 in the Cell-Wall Integrity of C. neoformans

In Candida albicans, the Cek1 MAPK, which is orthologous to Cpk1 in C. neoformans, is involved in the cell-wall biogenesis
(Roman et al., 2009). We assessed whether CPK1 deletion exacerbates the cell-wall integrity defects in cells deleted of Mpk1, which is the cell-wall integrity-regulating MAPK in $C$. neoformans, to prove that Cpk1 is involved in the cell-wall biogenesis. As reported earlier (Kraus et al., 2003), the mpk1 $\Delta$ mutant showed highly increased susceptibility to CFW and $\mathrm{CR}$, whereas the cpk1 $\Delta$ mutant did not (Figure 8). Notably,

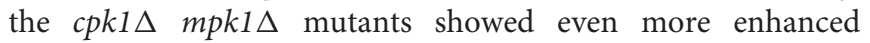
susceptibility to CFW and CR than the mpk1 $1 \Delta$ mutants (Figure 8), indicating that $\mathrm{Mpk} 1$ and Cpk1 play major and minor roles, respectively, in the cell-wall integrity. To assess the role of Sho1 and Msb2 in the cell-wall integrity, we also constructed the sho1 $\Delta$ mb2 $\Delta$ mpk1 $\Delta$ triple mutants in $C$. neoformans. The sho $1 \Delta$ msb2 $\Delta$ mpk $1 \Delta$ triple mutants were also more susceptible to CFW and CR than the mpk1 $\Delta$ mutants (Figure 8). Collectively, Sho1 and Msb2 contribute to cell wall biogenesis, along with Mpk1 and Cpk1, in C. neoformans.

The corrected Figure 8 appears below.

A correction has been made to the Discussion section, paragraph 1.

"This study for the first time proposed the regulatory mechanism of the Sho1-dependent and Msb2-dependent signaling pathways in C. neoformans. In addition, this study demonstrated that Shol is largely dispensable for the regulation of the HOG pathway for the osmoresistance, thermotolerance, and cryostress resistance. Instead, Shol plays Hog1-independent roles in the osmoresistance and thermotolerance. We also found that C. neoformans contains Msb2, which is the mucin-like TM Msb2 protein ortholog, known to interact with Sho1 in S. cerevisiae (Tatebayashi et al., 2007). However, while C. neoformans Msb2 and Sho1 appear to be colocalized in similar subcellular compartments, there is no evidence of 
their direct interactions. Supporting this, Sho1 and Msb2 play complementary, but distinct roles in biological responses of $C$. neoformans. Like Sho1, Msb2 contributes to the osmotolerance, cell membrane integrity, and cryostress resistance, but frequently not to the same extent, and is not markedly involved in regulation of Hog1 phosphorylation. Sho1 and Msb2 play also overlapping roles in the late stage of sexual differentiation, filamentous growth, in the Cpk1-independent manner. Furthermore, Cpk1, Sho1 and Msb2 contribute to cell wall biogenesis, along with Mpk1. However, during pulmonary infection in the mammalian host cryptococcal Msb2 and Sho1 roles are distinct. Msb2 promotes the acute adaptation to the host environment and seems to be dispensable thereafter. By contrast, Shol does not play a substantial role during the acute adaptation but it is required for the optimal fungal growth of fungus in the lungs during the later time points (Figure 9; Malachowski et al., 2016) where, as we demonstrated, it interferes with the development of the immune defenses."

A correction has been made to the Discussion section, paragraph 5.

"Despite the divergent function of Sho1 and Msb2 among fungi, their role in the filamentous growth and morphological differentiation seems evolutionarily conserved, although their regulatory mechanisms are rather different. This study suggests that Sho1 and Msb2 play a redundant role in promoting the filamentous growth of $C$. neoformans but does not regulate pheromone production during mating, which is well-known to be regulated by the Cpk1 MAPK pathway (Kss1 in $S$. cerevisiae and Cek1 in C. albicans). In C. albicans, however, Sho1 and Msb2 promote the filamentous growth and invasive growth by activating and phosphorylating the Cek1 MAPK (Roman et al., 2005). Likewise, Shol ortholog in C. lusitaniae is also known to be involved in the pseudohyphal development

\section{REFERENCES}

Boisnard, S., Ruprich-Robert, G., Florent, M., Da Silva, B., ChapelandLeclerc, F., and Papon, N. (2008). Role of Sholp adaptor in the pseudohyphal development, drugs sensitivity, osmotolerance and oxidant stress adaptation in the opportunistic yeast Candida lusitaniae. Yeast 25, 849-859. doi: 10.1002/yea.1636

Kraus, P. R., Fox, D. S., Cox, G. M., and Heitman, J. (2003). The Cryptococcus neoformans MAP kinase Mpk1 regulates cell integrity in response to antifungal drugs and loss of calcineurin function. Mol. Microbiol. 48, 1377-1387. doi: 10.1046/j.1365-2958.2003.03508.x

Lanver, D., Mendoza-Mendoza, A., Brachmann, A., and Kahmann, R. (2010). Sho1 and Msb2-related proteins regulate appressorium development in the smut fungus Ustilago maydis. Plant Cell 22, 2085-2101. doi: 10.1105/tpc.109.073734

Lee, K. T., So, Y. S., Yang, D. H., Jung, K. W., Choi, J., Lee, D. G., et al. (2016). Systematic functional analysis of kinases in the fungal pathogen Cryptococcus neoformans. Nat. Commun. 7:12766. doi: 10.1038/ncomms12766

Ma, Y., Qiao, J., Liu, W., Wan, Z., Wang, X., Calderone, R., et al. (2008). The sho1 sensor regulates growth, morphology, and oxidant adaptation in Aspergillus fumigatus but is not essential for development of invasive pulmonary aspergillosis. Infect. Immun. 76, 1695-1701. doi: 10.1128/IAI.01507-07

Malachowski, A. N., Yosri, M., Park, G., Bahn, Y. S., He, Y., and Olszewski, M. A. (2016). Systemic approach to virulence gene network analysis for gaining new insight into cryptococcal virulence. Front. Microbiol. 7:1652. doi: $10.3389 /$ fmicb. 2016.01652
(Boisnard et al., 2008). In S. cerevisiae, Shol serves as a receptor for the pseudohyphal growth pathway (O'Rourke and Herskowitz, 1998). In A. fumigatus, Shol also controls the hyphal development (Ma et al., 2008). In another basidiomycetous fungus, Ustilago maydis, Sho1 (UmSho1) also regulates the Cpk1like MAPKs, Kpp2 and Kpp6, both of which are required for the appressorium development and its function, although UmShol is not involved in mating and stress responses, implicating that UmShol is uncoupled to the HOG pathway (Lanver et al., 2010)."

A correction has been made to the Discussion section, paragraph 6.

"Although Sho1 and Msb2 do not regulate Cpk1-mediated pheromone production during mating, we found that the two proteins have a redundant role, along with Cpk1 and Mpk1, in regulating the cell-wall integrity in C. neoformans. The cpk1 $\Delta$ mutant does not show any increased susceptibility to cell-wall destabilizers, CFW and CR, and an ER stress agent TM (Lee et al., 2016), which is in stark contrast to the C. albicans cek1 $\Delta$ mutant displaying the increased sensitivity to cell-wall and ER stress agents (Roman et al., 2009). This study, however, reported that Cpk1, indeed, plays a minor role in the cell-wall biogenesis

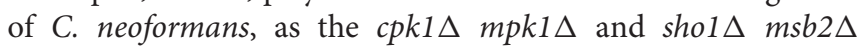
$m p k 1 \Delta$ mutants show a higher cell-wall integrity defect than the mpk1 $\Delta$ mutant; this finding indicates that $\mathrm{Cpk} 1$ and $\mathrm{Mpk} 1$ play redundant roles in the cell-wall biogenesis in C. neoformans, although the latter plays more dominant roles. Thus, this study is the first to report that Cpk1 is involved in the cell-wall biogenesis during the vegetative growth of $C$. neoformans, besides its known role in sexual differentiation.

The authors apologize for these errors and state that this does not change the main scientific conclusions of the article. The original article has been updated.

O'Rourke, S. M., and Herskowitz, I. (1998). The Hogl MAPK prevents cross talk between the HOG and pheromone response MAPK pathways in Saccharomyces cerevisiae. Genes Dev. 12, 2874-2886. doi: 10.1101/gad.12.1 $8.2874 \mathrm{c}$

Roman, E., Cottier, F., Ernst, J. F., and Pla, J. (2009). Msb2 signaling mucin controls activation of Cek1 mitogen-activated protein kinase in Candida albicans. Eukaryot. Cell 8, 1235-1249. doi: 10.1128/EC.00 081-09

Roman, E., Nombela, C., and Pla, J. (2005). The Shol adaptor protein links oxidative stress to morphogenesis and cell wall biosynthesis in the fungal pathogen Candida albicans. Mol. Cell. Biol. 25, 10611-10627. doi: 10.1128/MCB.25.23.10611-1062 7.2005

Tatebayashi, K., Tanaka, K., Yang, H. Y., Yamamoto, K., Matsushita, Y., Tomida, T., et al. (2007). Transmembrane mucins $\mathrm{Hkr} 1$ and Msb2 are putative osmosensors in the SHO1 branch of yeast HOG pathway. EMBO J. 26, 3521-3533. doi: 10.1038/sj.emboj.7601796

Copyright (C) 2020 So, Jang, Park, Xu, Olszewski and Bahn. This is an open-access article distributed under the terms of the Creative Commons Attribution License (CC BY). The use, distribution or reproduction in other forums is permitted, provided the original author(s) and the copyright owner(s) are credited and that the original publication in this journal is cited, in accordance with accepted academic practice. No use, distribution or reproduction is permitted which does not comply with these terms. 